\title{
(a) A Novel Microsurgical Rodent Model for the Transplantation of Engineered
} Cardiac Muscle Flap

(b) Authors:

Richard Tee, MBBS, $\mathrm{PhD}^{1,2}$

Wayne Allan Morrison, MBBS, MD, FRACS ${ }^{1,2 *}$

Rodney J. Dilley $\mathrm{PhD}^{1,2,3}$

\section{(c) Affiliations:}

1 O'Brien Institute, 42, Fitzroy Street, Fitzroy, Victoria, 3065

${ }^{2}$ Department of Surgery, University of Melbourne, Victoria, 3065

${ }^{3}$ School of Surgery, University of Western Australia, Nedlands, Western Australia, 6009, Australia

(d)

Grant sponsors: The National Health and Medical Research Council

Grant number: 509271

(e) Correspondence to:

Dr Richard Tee

Address: Hutt Hospital, High Street, Lower Hutt, 5010

Email: richardteembbs@gmail.com

Telephone: +64-4-5666999

Fax: N/A

(f) Running title: Rat Cardiac Flap Transplant Model

This is the author manuscript accepted for publication and has undergone full peer review but has not been through the copyediting, typesetting, pagination and proofreading process, which may lead to differences between this version and the Version of record. Please cite this article as doi:10.1002/ micr.30325. 


\section{Abstract}

Background: The survival of engineered cardiac muscle 'grafts' to the epicardium is limited by vascularization post-transplantation in rat models. In this paper we describe the methodology of a novel rat model that allows for the transplantation of an engineered cardiac muscle flap (ECMF) onto the epicardium.

Materials and Methods: A total of 40 rats were used. 24 neonatal rats were used to harvest cardiomyocytes. At week 1, ECMF were generated by seeding cardiomyocytes into the arteriovenous loop (AVL) tissue engineering chamber implanted into the right groin of adult rats $(n=8)$. At week 6 , the ECMF were harvested based on a pedicle along the femoral-iliac-abdominal vessel and anastomosed to the neck vessels of the recipient syngeneic adult rats $(n=8)$. The flaps were delivered into the thoracic cavity and onto the epicardium. The transplanted flaps were harvested at week 10. Survival of the flaps was assessed by the patency of anastomoses and viability of the cardiomyocytes through histological analysis (hematoxylin and eosin, desmin, vWF immunostaining).

Results: 6 out of 8 rats survived the transplantation procedure. These remaining 6 recipient rats survived until harvest time point at four weeks post-transplantation. The mean area of the flap was $46.7 \mathrm{~mm}^{2} .6$ out of 6 flaps harvested at week 10 showed viable cardiomyocytes using desmin immunostaining and vascular channels were seen at the interface between flap and epicardium.

Conclusion: This is a technically feasible model that will be useful for future assessment of different cardiac stem cell implants and their functional significance in rat heart models.

Keywords: Cardiac, Tissue Engineering, Rat Model, Transplantation, Microsurgery 


\section{INTRODUCTION}

Various techniques for cardiac tissue 'graft' engineering in vitro were described in the literature $^{1}$. Several groups have reported the successful implantation of these avascular cardiac grafts onto the epicardium in animal studies ${ }^{2-6}$. Zimmermann et $\mathrm{al}^{5}$ reported significant improvement in cardiac function after the transplantation of tissue engineered cardiac grafts onto diseased myocardium post-myocardial infarction. However, the report of cardiomyocyte survival post-grafting has been poor ${ }^{7}$. The survival of these grafts relies on the process of imbibition, inosculation and subsequently angiogenesis. Unlike skin or cartilage, these functional cardiac grafts have high metabolic demands, and are more prone to ischemic injury whilst the process of 'graft take' occurs. Furthermore, laying grafts onto ischemic myocardium seems counterintuitive to traditional surgical teaching.

A different approach for tissue engineering was proposed by Mian et al using in vivo vascularization technique in an arteriovenous loop (AVL) tissue engineering chamber $^{8}$. This technique allows the implantation process to take place in a well vascularized tissue bed prior to assembly into a metabolic demanding contractile tissue. Morritt et al. adapted this technique in cardiac tissue engineering ${ }^{9}$ and successfully reported the assembly of neonatal cardiomyocytes into a potentially transplantable flap in nude rats $^{9}$. It was the thickest cardiac tissue generated to date.

While the potential of the engineered cardiac muscle flap (ECMF) was reported by its transplantation to the contralateral groin, a model that delivers the ECMF to the native environment of the heart for further functional study was lacking as there were no existing model that allow such transplantation.

In our previous study, we demonstrated there was no muscle volume loss after 
transplantation of the ECMF to an ectopic site as well as the feasibility of transplanting the ECMF onto the epicardium ${ }^{10}$. However, the technical aspects of the transplantation model was not described.

This article describes the technical details and feasibility of the novel microsurgical rodent model for transplanting an ECMF to the epicardium of syngeneic rats. This model will provide a platform for assessing cell survival and the potential of the cardiac muscle flap as a bioventricular assisting device.

\section{MATERIALS AND METHODS}

All procedures were performed with the approval of the St Vincent's Hospital Animal Ethics Committee and conformed to National Health and Medical Research Council (Australia) guidelines for animal welfare. A total of 40 Sprague Dawley rats were used in the experiment (including 24 neonatal rats and 16 adult rats).

\subsection{Preparation of neonatal cardiomyocytes}

Neonatal rat cardiomyocytes were isolated from syngenic neonatal Sprague Dawley rats ( $\mathrm{n}=24,1-3$ days old; Experimental Medicine and Surgery Unit, St. Vincent's Hospital Melbourne, Australia). 3 neonatal rats were required to isolate $6 \times 10^{6}$ cardiomyocytes sufficient for a single chamber implantation (thus, 8 chambers required 24 neonates). Cardiomyocytes required for the initial implantation were isolated enzymatically from the ventricles removed from the neonates, beginning from the night before implantation, as previously described ${ }^{10} .6 \times 10^{6}$ rat viable cardiomyocytes counted in a hemocytometer were suspended in $50 \mu$ of cardiomyocyte medium (DMEM/F12 with HEPES, 5\% horse serum, 1\% antibiotic/antimycotic solution supplemented by $3 \mathrm{mM}$ pyruvic acid, $2 \mathrm{~g} / \mathrm{L}$ bovine 
serum albumin, $100 \mathrm{mg} / \mathrm{mL}$ ampicillin, $4 \mathrm{mg} / \mathrm{mL}$ transferrin, $0.7 \mathrm{ng} / \mathrm{mL}$ sodium selenite, $5 \mathrm{mg} / \mathrm{mL}$ linoleic acid, and $100 \mathrm{mM}$ ascorbic acid) mixed in $200 \mu \mathrm{L}$ hydrogel (Matrigel ${ }^{\mathrm{TM}}$, BD Biosciences Pharmingen) and kept on ice until implantation.

The transplantation model involved using a donor rat for the in vivo engineering of ECMF (described in section 2.2) and transplantation based on a long pedicle that includes the femoral, iliac and abdominal vessels (described in section 2.3). The pedicle was anastomosed to the left cervical vessels and delivered to a recipient rat's heart as depicted in Figure 1.

\subsection{Engineering of Cardiac Muscle Flap}

An AVL tissue engineering chamber was constructed in the right groin of syngeneic adult Sprague Dawley rats ( $\mathrm{n}=8$, weight $250-350$ grams) intended as donors as previously described ${ }^{9,11}$. In brief, the femoral artery and vein in the right groin were isolated, separated and ligated distally. A contralateral femoral vein graft was harvested from the contralateral groin and interposed between the previously isolated femoral artery and vein. The microanastomoses were performed using 10-0 nylon to form the AVL. The AVL was placed within a polycarbonate chamber $(0.5 \mathrm{~mL}$ internal volume, $1.3 \mathrm{~cm}$ internal diameter, and $0.5 \mathrm{~cm}$ height; Department of Chemical Engineering, The University of Melbourne, Melbourne, Australia). The chamber was then secured to the inguinal ligament and surrounding soft tissues with 5-0 polypropylene sutures through perforations on the base of the chamber. The cardiomyocyte suspension was then pipetted onto the AVL and the gel solidified as it reached $37^{\circ} \mathrm{C}$. Finally, the lid of the chamber was applied and the skin wound was closed. No immunosuppressant was required for the syngeneic rats, thus avoiding undesirable effects of the immunosuppressant. 


\subsection{Engineered Cardiac Muscle Flap Transplantation}

At 6 weeks post-implantation of the cardiomyocytes, AVL chambers were explored in the donor rats. This time point was selected based on a previous study ${ }^{9}$, in which a 4 week ECMF was the earliest time point the flap was observed to spontaneously contract. A slightly later time point of six weeks was used to allow for maturation of the flap and more reliable contractility for transplantation.

The polycarbonate chamber was removed to reveal the contractile ECMF. The pedicle of the ECMF was exposed by careful dissection to remove encapsulating fibrous tissue.

Without dissecting the pedicle further, a midline laparotomy incision was then made and the incision was extended to the groin wound. The small intestine was carefully externalized and wrapped in moist gauze. A moist cotton bud was then used to tease away intraperitoneal tissues until the abdominal aorta (AA) and inferior vena cava (IVC) and their bifurcation into left and right common iliac vessels were visualized.

Further dissections were performed until the common iliac vessels were seen entering the groin. The abdominal wall that separates the iliac vessels and the pedicle of the flap (the superficial femoral vessels) was carefully divided to free the pedicle. This step was the most technically challenging step of the procedure as there were many emanating branches from the femoral vessels and would best be done under an operating microscope. The final step would be the ligation of the deep femoral branch.

After freeing the ECMF pedicle from the groin, sacral and deep spinal branches of the posterior abdominal wall were carefully ligated from caudal to cranial. The dissection 
was stopped at the level of the hepatic branches (Figure 2), cranial to the renal branches (Figure 1). The renal vessels were not ligated at this point to maintain the clearance of metabolic waste from the flap. Once the pedicle of the ECMF was completely freed, except from the renal and hepatic branches, the peritoneal cavity was packed with warm saline gauze and attention turned to the recipient rat.

The recipient rats ( $\mathrm{n}=6$, weight 407-510 grams) were anaesthetized with inhalational anaesthesia (1-2\% Halothane, AstraZeneca Pty Ltd, NSW, Australia) in a gas chamber, followed by intubation with a 14-gauge cannula. Mechanical ventilation of the animal was kept at the settings of $1.5-2 \%$ of halothane and ventilation rate of 90 stroke per min utilizing a small animal ventilator (Model 683 Small Animal Ventilator, Harvard Apparatus). The recipient animal was kept on the mechanical ventilator until the closure of the chest wound.

A midline incision in the neck was made with the incision extending to the left caudally to expose the left sided chest wall of the rat. The jugular vein which lies right under the skin was identified and dissected free of surrounding tissue at this point. The vein was dissected as distal from its draining vein as possible. Whenever a branching point was seen, the vein was ligated at the branching point to allow for a larger lumen for anastomosis.

Midline muscles were retracted using sutures and with gentle dissection using the cotton bud, the left common carotid artery was exposed. A ligating clip was placed as cranial as possible to obtain maximum length of the artery for subsequent anastomosis. Both the artery and vein were placed in position with vessel clamped and prepared for anastomosis.

Returning to the donor rat, ligation of the AA and IVC was performed. Immediately 
after ligation, a 16 gauge cannula was used to flush $4{ }^{\circ} \mathrm{C}$ heparinised saline through the AA until clear fluids were seen coming out of IVC. The ECMF was wrapped with gauze soaked in the cold heparinized saline and deliver over to the operating table of the recipient rat.

End-to-end anastomoses of the AA to carotid artery and IVC to jugular vein were performed respectively under operating microscope magnification. The flap was kept moist wrapped in gauze at $4{ }^{\circ} \mathrm{C}$ throughout the procedure. After the anastomoses were sutured, the vessel clamps were released and ECMF immediately perfused. The ECMF would then slowly resume contraction (all ECMF resumed contraction before progressing to thoracotomy).

Following this, standard anterior thoracotomy was then performed (Figure 3A). The location of the heart is at the $5^{\text {th }}$ and $6^{\text {th }}$ intercostal space, or it can be identified by palpation. An artery forceps was used to bluntly dissect through the chest wall into the thoracic cavity. The pericardial sac and fat on the surface of the epicardium were gently removed with a moist cotton bud to expose the epicardium.

Finally, the capsular surface of the flap that faced the epicardial surface of the flap was excised. Following this, the flap was delivered into the chest to lay on the epicardium (Figure 3B). Once the position of the flap was decided, three interrupted 7-0 Prolene sutures were used to anchor the remaining fibrous edges to the epicardium to allow myocardium to epicardium contact. This step was most conyeniently performed under $2.5 x$ loupe magnification. A segment of rib was removed to prevent a kink of the pedicle as required. The chest was then closed in layers, including loosely closing the split musculature (Figure 3C).

\subsection{Assessments}


4 weeks post-transplantation the tissues were harvested en bloc with the heart. The anatomoses at the neck were first exposed to check for patency before the harvest of the heart tissue. Recipient animals were sacrificed at this time point. Success of the procedure was defined by the survival of the animal after successful transplantation up to the 4-week time point, and upon exploration maintained a patent anastomosis with survival of the cardiomyocytes contained within the flap. Success rate was, thus, the number of success with the transplantation procedure over the total number of rats used. Flap survival was defined by the presence of cardiomyocytes within the flap post-transplantation.

$5 \mu \mathrm{m}$ thick paraffin sections were made and stained with hematoxylin and eosin (H\&E) for general histology. Survival of cardiomyocytes were detected using desmin immunostaining (1:100, Dako). Blood vessels running between the heart and flap were assessed using Von Willebrand Factor (vWF, 1:200, Millipore) immunostaining. $\longrightarrow$

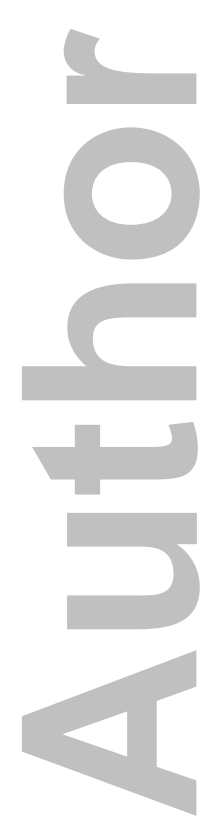




\section{RESULTS}

6 out of 8 animals had successful procedures (success rate $75 \%$ ). The approximate cold ischemic time of the flap was 40 minutes. 2 animals died during the transplantation procedure so were considered technical failures. The first animal had uncontrolled intraoperative bleed from a ruptured superficial cardiac vein. The second animal had an intraoperative thrombosis at the anastomosis site that was not salvageable.

All animals $(n=6)$ that had successful transplantation survived through four weeks post-surgery, with no signs of irritability. All 6 animals had patent anastomoses four weeks post-surgery, demonstrated by positive patency tests at the arterial and venous anastomoses. All ECMF were found attached to the epicardium at the time of harvest (Figure 4). The mean area of the flap was $46.7 \mathrm{~mm}^{2}$ (range $37.5-60, \mathrm{~mm}^{2}$ ), which occupied approximately two thirds of the anterior surface of the heart.

Desmin immunostaining confirmed the survival of the cardiomyocytes after transplantation. The cardiomyocytes tended to locate closer to the vascular lumen of the AVL-based flap (Figure 5). In two specimens, the cardiac muscles were observed to lie in proximity to the interface between the flap and heart. However, there was no visible integration of the transplanted cardiac muscle and host myocardium, the muscle cells were instead separated by a layer of connective tissue where the ECMF capsule and epicardium merged (Figure 6). There were vessels running through the epicardial connective tissue of the host heart and also in the connective tissue of the flap (Figure 7). There was no demonstrable immune response to the flap as demonstrated by $H \& E$ histology with generally clear connective tissue with no significant lymphocytic or macrophage cellular infiltrate which would manifest as 


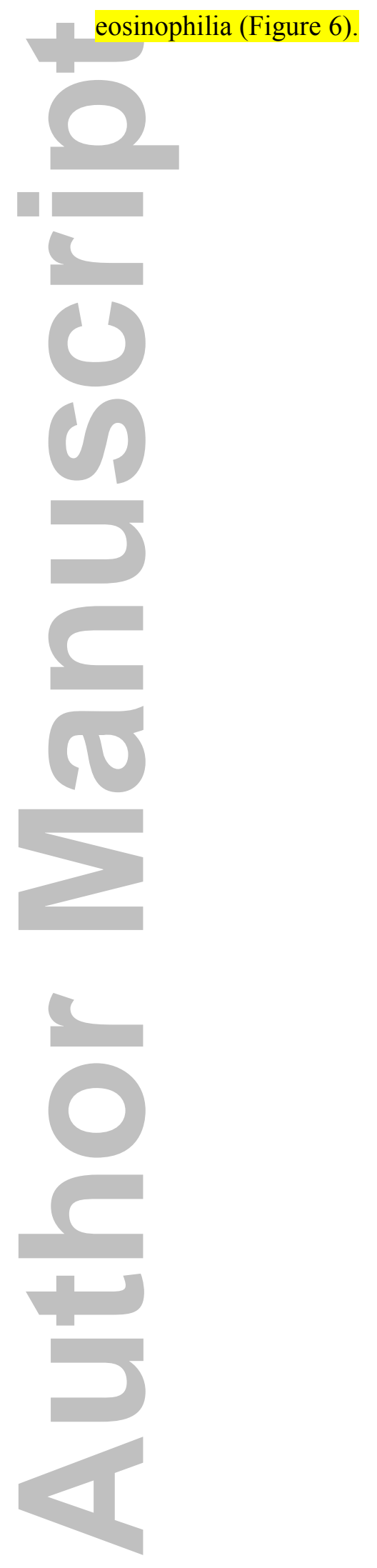

John Wiley \& Sons

This article is protected by copyright. All rights reserved. 


\section{DISCUSSION}

We have shown previously, using an ectopic transplant model to the neck vessels, the transplant process do not affect the volume of cardiac tissue transplanted ${ }^{10}$. In this article, we described, in detail, the methodology for a proof-of-concept model for the free transfer of engineered vascularized cardiac muscle to the epicardium in a syngeneic rat model. We demonstrated the feasibility of this novel small animal model by a microsurgeon.

Despite progress made in two decades of cardiac tissue engineering, a robust vascularized cardiac tissue that is ready for transplantation is still lacking. There were many techniques utilized that managed to successfully create contractile cardiac tissue grafts ${ }^{1}$. These techniques, however, shared a common strategy of seeding isolated neonatal rat cardiomyocytes into some form of scaffold ${ }^{3,12,13}$. One of the landmark studies by Zimmermann et $\mathrm{al}^{5}$ demonstrated the transplantation of avascular engineered heart tissue (EHT) grafts onto the epicardium of diseased hearts in syngeneic rats. Using sophisticated assessment tools, they were able to show EHT integrated electrophysiologically with the heart and improved cardiac function after induced myocardial infarction. While several research groups have demonstrated in vitro generated cardiac muscle grafts transplanted to failing heart in rodent models to improve heart function, there seems to be significant loss of cardiomyocytes post-

transplantation $^{7}$. There appears to be an underestimation of the importance of vascularization of these grafts.

The importance of vascularization of the engineered cardiac grafts was gradually recognized to improve its survival. Studies by Suzuki et $\mathrm{al}^{14}$ and Dvir et al ${ }^{15}$ showed independently, that cardiac grafts generated in vitro followed by prevascularisation by 
the omentum (cardiac only grafts placed in the omentum to allow penetration of vessels and hence formation of vascular network within the construct) prior to or at transplantation demonstrate greater improvement of cardiac function in the recipient rats suffering from myocardial infarction. It appears that contractile engineered grafts, seemed to have an effect on improving injured hearts and prevascularisation of the in vitro generated constructs seemed to improve the functions of these grafts. It was speculated that this observation was related to the improved survival of cardiomyocytes within the grafts. However, as clinicians, it is clear that this improvement is not adequate to allow clinical translation of its use.

The expectation of cardiac muscle grafts to 'take' like skin grafts on a hostile environment of infarcted myocardium (suboptimal wound bed) seemed counterintuitive clinically. Taking a different approach, Morritt et $\mathrm{al}^{9}$ successfully engineered contractile cardiac muscle flaps using the AVL tissue engineering chamber. Unlike previous techniques utilizing in vitro assembly of cardiomyocytes onto a scaffold with subsequent transplantation, the AVL tissue engineering chamber utilizes an in vivo tissue engineering technique. This technique allows cardiomyocyte assembly to occur in vivo around a microsurgically created vascular system, with arterial in flow and venous outflow. The cardiomyocyte lies within a highly angiogenic environment ${ }^{16}$ prior to its assembly (low metabolic state) and subsequent formation into a flap with a transplantable pedicle.

While in principle, the ECMF can then be delivered to the heart and immediately be revascularised, the functional effect of the ECMF on the heart needs further elucidation. Furthermore, to investigate the effect of the cardiac muscle flap on injured or failing heart, a model that delivers the flap to the epicardium is required. 
Ideally, an autologous transplantation from the chamber in the groin to the epicardium would be preferred.

In the process of developing the model, we explored the deep inferior epigastric vessels on the contralateral side to the chamber and the thoracodorsal vessels in an attempt to design a true autologous transplant model. However, mismatch of these vessels to the femoral vessels was too great. While the neck vessels are a better size match, tunneling the flap through the thoracic inlet in rats was proved not feasible during our dissection. After failed attempts in generating an autologous transfer model, we shift our focus to utilizing the convenience of having a syngeneic rat model.

Our initial idea was to adopt the abdominal heterotopic rat transplant model from immunology transplant studies ${ }^{17}$. However, no matter how caudally we performed the anastomosis to place the transplanted heart closer to the groin, the ECMF will not reach the transplanted heart without devascularizing the hind limb. Thus, the new idea of using the donor rat to harvest a flap with a long pedicle that extends into the abdominal vessels was conceived. While there were initial concerns of size mismatch when using of abdominal vessel for anastomosis to the neck vessels, end-to-end anastomosis appears to give the best outcomes. Attempts to perform end-to-side anastomosis or utilizing the more size matching renal vessels had been shown to be more likely to kink and twist resulting in thrombosis of the ECMF pedicle. This model described allowed a generous pedicle and less insult to the recipient animal. It also allowed a more physiological heart compared to an abdominal heterotopic rat heart model $^{17}$.

Technically, the dissection of the pedicle through the abdominal wall into the groin 
was most challenging, with many branching vessels caudal to the common iliac vessels arising within the abdominal wall musculature. A viable animal is required prior to the transplant, therefore, uncontrolled blood loss during any part of the dissection is highly undesirable. Another technical challenge would be the inset of the ECMF onto the epicardium. A shallow bite using the suture needle on the surface of the heart can tear through rupturing the cardiac veins, while a relatively deep bite when incorrectly placed can induce an infarct by ligating the descending (LAD) coronary artery. Reviewing histological cross section of the rat heart (noting the thickness), understanding the anatomy of the LAD artery and practicing suturing fat pad on the epicardium in practice animals will help greatly. The two failures came during the initial four animal surgeries. The final four animal surgeries were uncomplicated. This suggests a learning curve that can be easily overcome with experience. Despite this, we achieved an overall success rate of $75 \%$

All the cardiac flaps remained attached at the time of exploration. While no direct contact of the host myocardium and flap's cardiac muscle was observed, higher density of vessels were observed at the border of contact on both the cardiac and flap side. Some of these vessels seemed to be running from the heart substance into the connective tissue layer of the flap suggesting there might be communication between the two circulations. Further perfusion studies would have to be conducted to investigate this observation.

A cardiac muscle flap is a functional flap rather than a filler flap, its ultimate goal is to function like the myocardium. A historical procedure dynamic latissimus dorsi cardiomyoplasty ${ }^{18}$, aims to improve failing heart function by acting as a bioventricular assisting device. However, despite preconditioning the latissimus dorsi 
flap before applying to the heart, the outcome of the procedure remained dismal. Using the ECMF, a flap composed of cardiac muscle, it should theoreticallly be more suitable for pacing. Our model will allow cardiac progenitor/stem cells that can assemble within the AVL chamber to be transplanted to the heart for functional assessment. In fact, our group has investigated adipose-derived stem cells ${ }^{19}$ and induced pluripotent stem (iPS) cells ${ }^{20}$ as cell sources for cardiac tissue engineering. Furthermore, in the well-established rat myocardial infarction model ${ }^{21}$, the anterior wall infarct generated by LAD ligation is precisely where the flap can conveniently be placed. Functional assessment of the heart is also possible using echocardiography or using intracardiac catheter ${ }^{5}$. The 4 -week time point, which we chose to harvest the transplanted cardiac flap, was selected in anticipation of future study when ligation of the left anterior descending coronary artery to induce myocardial infarction would be included in the model. A 4-week time point was thought to allow more significant dilated cardiomyopathy to be detected by echocardiography. Assessment during the four weeks for the patency of the pedicle was deemed not necessary as we do not believe that the cardiomyocytes would be able to survive should the pedicle be obstructed. Cardiomyocytes are highly metabolically demanding cells. If the pedicle were to thrombosed during the four weeks after transplantation and recanalized subsequently before harvest, the cardiomycytes would have no chance of survival. Particularly, when considering the cardiomyocytes are closer to the AVL (Figure 5)

rather than the epicardium preventing diffusion of nutrients from the surface of the heart.

Whilst the surgery is labor intensive, this microsurgical syngeneic rat model is a necessary platform for the future assessment of engineered cardiac muscle flaps. 


\section{CONCLUSIONS}

The paper demonstrated a rat model that has significant implications for the advancement of cardiac tissue engineering using the AVL tissue engineering chamber.

\section{ACKNOWLEDGEMENTS}

We thank Sue Mackay, Liliana Pepe, Anna Deftereos, Amanda Rixon, Priya

Sivakumaran, Jason Palmer, Xiao Lian Han for their technical assistance.

\section{DISCLOSURE STATEMENT}

Professor Morrison is an inventor on the Vascularized Tissue Graft patent and entitled to proceeds derived from commercialization of the patent.

\section{REFERENCES}

1. Tee R, Lokmic Z, Morrison WA, Dilley RJ. Strategies in cardiac tissue engineering. ANZ J Surg 2010;80(10):683-693.

2. Furuta A, Miyoshi S, Itabashi Y, Shimizu T, Kira S, Hayakawa K, Nishiyama N, Tanimoto K, Hagiwara Y, Satoh T, Fukuda K, Okano T, Ogawa S. Pulsatile cardiac tissue grafts using a novel three-dimensional cell sheet manipulation technique functionally integrates with the host heart, in vivo. Circ Res 2006;98(5):705-712.

3. Leor J, Aboulafia-Etzion S, Dar A, Shapiro L, Barbash IM, Battler A, Granot Y, Cohen S. Bioengineered cardiac grafts: A new approach to repair the infarcted myocardium? Circulation 2000;102(19 Suppl 3):III56-61.

4. Lesman A, Habib M, Caspi O, Gepstein A, Arbel G, Levenberg S, Gepstein L. Transplantation of a tissue-engineered human vascularized cardiac muscle. Tissue Eng Part A 2010;16(1):115-125.

Zimmermann WH, Melnychenko I, Wasmeier G, Didie M, Naito H, Nixdorff U, Hess A, Budinsky L, Brune K, Michaelis B, Dhein S, Schwoerer A, Ehmke $\mathrm{H}$, Eschenhagen T. Engineered heart tissue grafts improve systolic and diastolic function in infarcted rat hearts. Nat Med 2006;12(4):452-458. 
6. Stevens KR, Kreutziger KL, Dupras SK, Korte FS, Regnier M, Muskheli V, Nourse MB, Bendixen K, Reinecke H, Murry CE. Physiological function and transplantation of scaffold-free and vascularized human cardiac muscle tissue. Proc Natl Acad Sci U S A 2009;106(39):16568-16573.

7. Zhang M, Methot D, Poppa V, Fujio Y, Walsh K, Murry CE. Cardiomyocyte grafting for cardiac repair: graft cell death and anti-death strategies. J Mol Cell Cardiol 2001;33(5):907-921.

8. Mian R, Morrison WA, Hurley JV, Penington AJ, Romeo R, Tanaka Y, Knight KR. Formation of new tissue from an arteriovenous loop in the absence of added extracellular matrix. Tissue Eng 2000;6(6):595-603.

9. Morritt AN, Bortolotto SK, Dilley RJ, Han X, Kompa AR, McCombe D, Wright CE, Itescu S, Angus JA, Morrison WA. Cardiac tissue engineering in an in vivo vascularized chamber. Circulation 2007;115(3):353-360.

10. Tee R, Morrison WA, Dusting GJ, Liu GS, Choi YS, Hsiao ST, Dilley RJ. Transplantation of engineered cardiac muscle flaps in syngeneic rats. Tissue Eng Part A 2012;18(19-20):1992-1999.

11. Zhan W, Marre D, Mitchell GM, Morrison WA, Lim SY. Tissue Engineering by Intrinsic Vascularization in an In Vivo Tissue Engineering Chamber. J Vis Exp 2016(111).

12. Li RK, Jia ZQ, Weisel RD, Mickle DA, Choi A, Yau TM. Survival and function of bioengineered cardiac grafts. Circulation 1999;100(19 Suppl):II63-69.

13. Eschenhagen T, Zimmermann WH. Engineering myocardial tissue. Circ Res 2005;97(12):1220-1231.

14. Suzuki R, Hattori F, Itabashi Y, Yoshioka M, Yuasa S, Manabe-Kawaguchi H, Murata M, Makino S, Kokaji K, Yozu R, Fukuda K. Omentopexy enhances graft function in myocardial cell sheet transplantation. Biochem Biophys Res Commun 2009;387(2):353-359.

15. Dvir T, Kedem A, Ruvinov E, Levy O, Freeman I, Landa N, Holbova R, Feinberg MS, Dror S, Etzion Y, Leor J, Cohen S. Prevascularization of cardiac patch on the omentum improves its therapeutic outcome. Proc Natl Acad Sci U S A 2009;106(35):14990-14995.

16. Lokmic Z, Stillaert F, Morrison WA, Thompson EW, Mitchell GM. An arteriovenous loop in a protected space generates a permanent, highly vascular, tissue-engineered construct. FASEB J 2007;21(2):511-522.

17. Amir G, Miller L, Shachar M, Feinberg MS, Holbova R, Cohen S, Leor J. Evaluation of a peritoneal-generated cardiac patch in a rat model of heterotopic heart transplantation. Cell Transplant 2009;18(3):275-282.

18. Chachques JC, Grandjean PA, Carpentier A. Latissimus dorsi dynamic cardiomyoplasty. Ann Thorac Surg 1989;47(4):600-604.

19. Choi YS, Matsuda K, Dusting GJ, Morrison WA, Dilley RJ. Engineering cardiac tissue in vivo from human adipose-derived stem cells. Biomaterials 2010;31(8):2236-2242.

20. Lim SY, Lee DG, Sivakumaran P, Crombie D, Slavin J, Dottori M, Conley B, Denham M, Leung J, Tee R, Dusting GJ, Pebay A, Dilley RJ. In vivo tissue engineering chamber supports human induced pluripotent stem cell survival and rapid differentiation. Biochem Biophys Res Commun 2012;422(1):75-79. 

21. Huang NF, Sievers RE, Park JS, Fang Q, Li S, Lee RJ. A rodent model of
myocardial infarction for testing the efficacy of cells and polymers for
myocardial reconstruction. Nat Protoc 2006;1(3):1596-1609. 


\section{Figure Legends}

Figure 1 Schematic diagram showed the strategy of isograft orthotopic transplantation of the engineered cardiac muscle flap by dissecting the pedicle of the flap along the femoral-iliac-abdominal vessel axis and connect it to the neck vessels of the recipient rat.

Figure 2 Cardiac muscle flap dissected through the groin to the level of the hepatic branch of the abdominal vessels in the donor rat (right). After end-to-end anastomosis of the abdominal aorta (AA) to the carotid artery and the inferior venae cavae (IVC) to the jugular vein (left) in the recipient rat.

Figure 3 (A) Cardiac muscle flap (dashed arrow) shown to lie over the chest wall with a thoracotomy window that exposed the anterior surface of the recipient's heart, (B) sutured to the heart through a thoracotomy window (note: a segment of the rib was resected to allow the entrance of the pedicle) and (C) The pedicle (solid arrow) ran from the neck vessels, through the split pectoral muscles into the thoracotomy window. 'Cranial' was marked to show orientation. IC=intercostal.

Figure 4 (A) Diaphragmatic approach to harvest the flap $\left(^{*}\right) \&$ heart to avoid damage to the pedicle (arrows) prior to the harvest (B) Macroscopic appearance of flap attached to the anterior surface of the ventricle.

Figure 5 Macroscopic section of harvested specimen (left) showed cross section of transplanted flap on heart surface, with the two lumens being the artery (smaller) 
and vein (larger) of the arteriovenous loop. Micrograph sections (right) showed 10x magnification desmin immunostained cardiomyocytes assembled close to the lumen of the flap that remained viable after transplantation. (scale bar=200 $\mu \mathrm{m})$

Figure 6 Representative micrographs from consecutive sections at the region of contact between the cardiac muscle flap and the rat heart (left) showed cardiac muscle tissue in the patch (dotted line) separated from the host myocardium by integrated connective tissue of the epicardium and the flap capsule. Picture (right) showed vWF immunostained vessel (arrow) coursed in the epicardium and into the connective tissue between the flap and the heart's surface. $v W F=$ von Willebrand factor. (scale bar $=200 \mu \mathrm{m})$

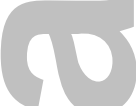

Figure 7 Representative micrographs of H\&E staining showed no demonstrable immune response at (A) the flap-heart interface and (B) within the flap. (scale

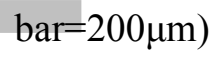

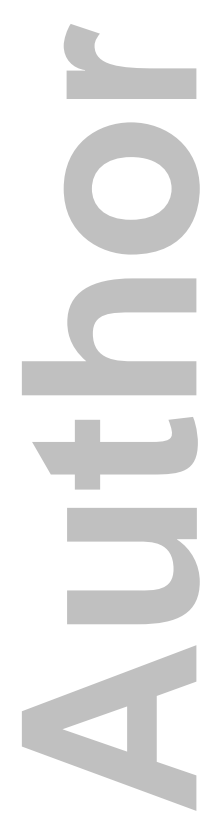




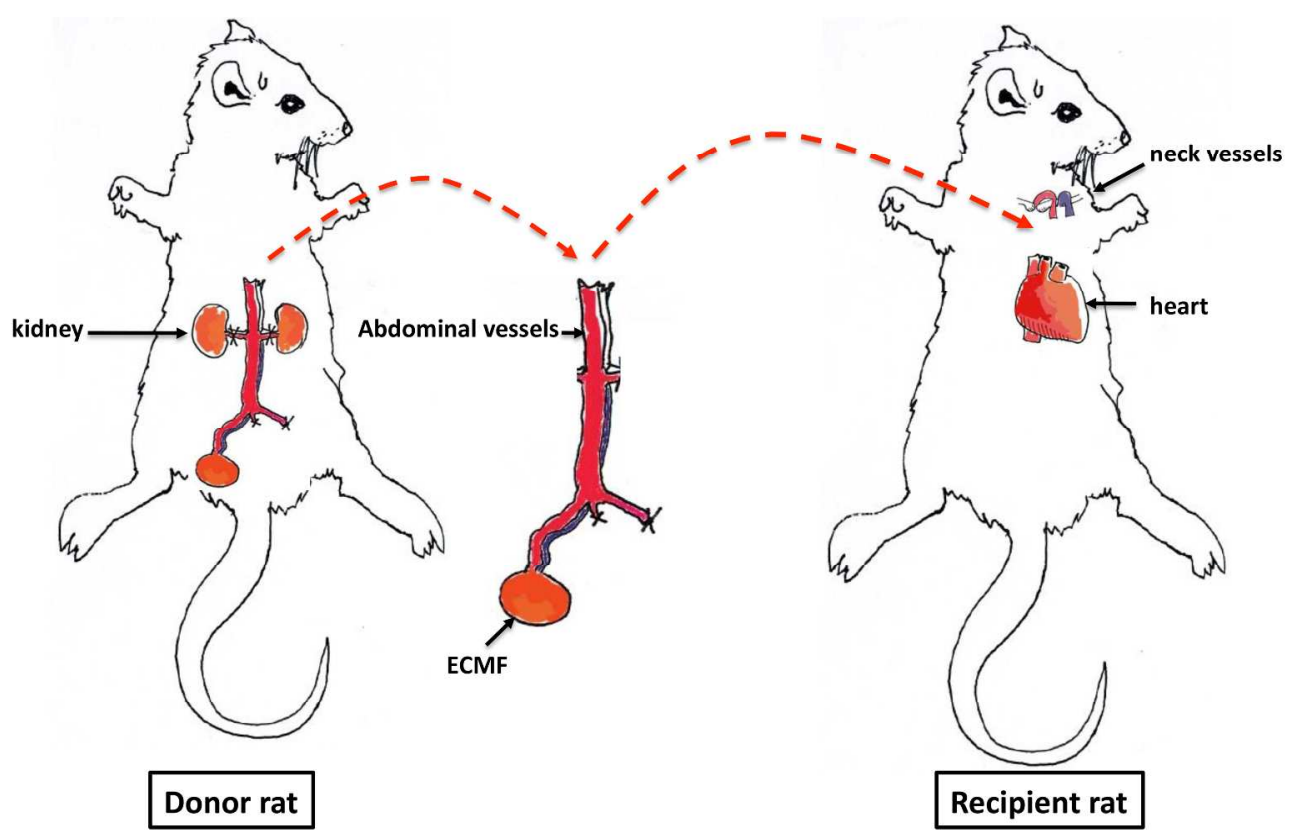

Figure 1 Schematic diagram showed the strategy of isograft orthotopic transplantation of the engineered cardiac muscle flap by dissecting the pedicle of the flap along the femoral-iliac-abdominal vessel axis and connect it to the neck vessels of the recipient rat.

$245 \times 154 \mathrm{~mm}(300 \times 300 \mathrm{DPI})$ 

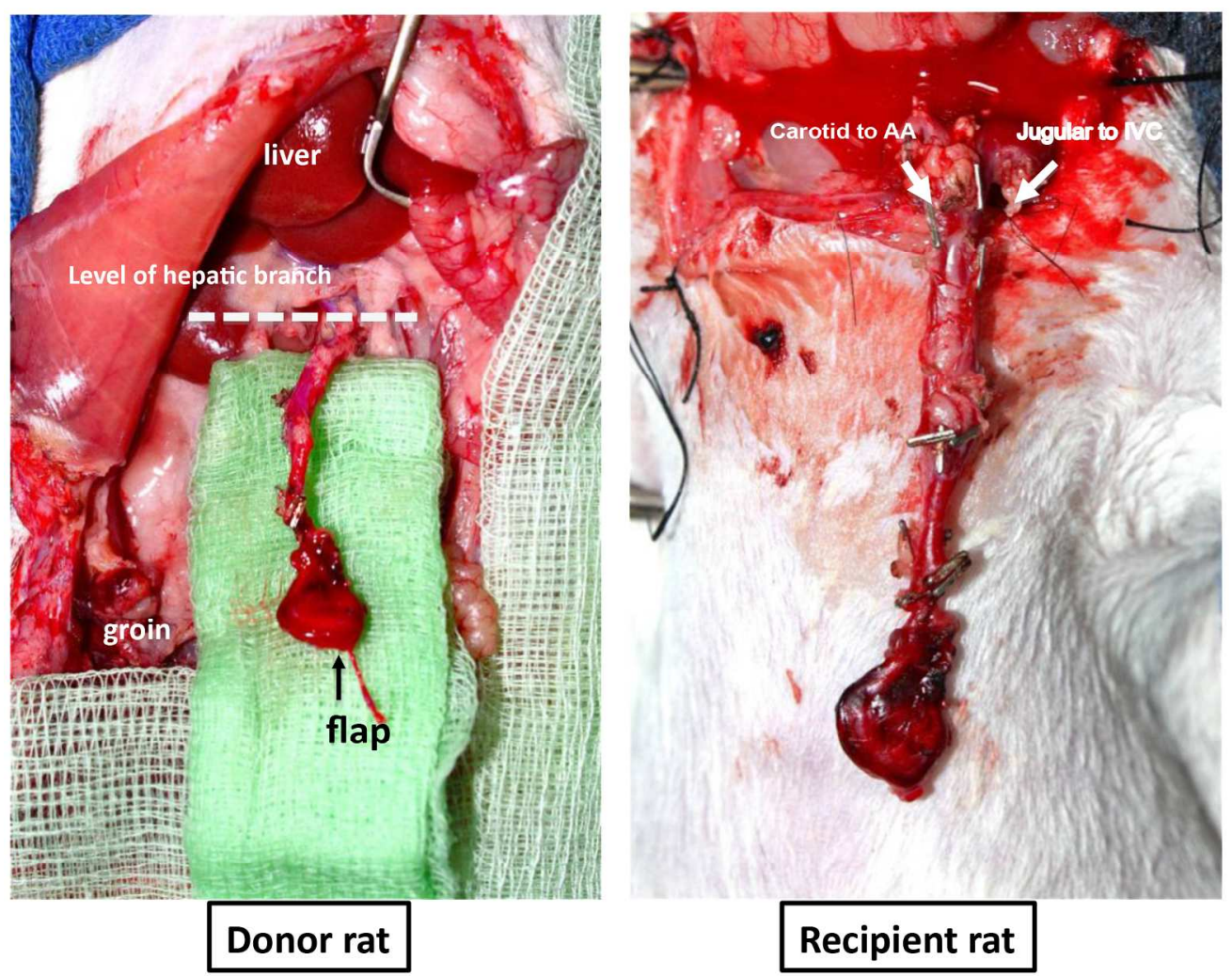

Figure 2 Cardiac muscle flap dissected through the groin to the level of the hepatic branch of the abdominal vessels in the donor rat (right). After end-to-end anastomosis of the abdominal aorta (AA) to the carotid artery and the inferior venae cavae (IVC) to the jugular vein (left) in the recipient rat.

$188 \times 150 \mathrm{~mm}(300 \times 300 \mathrm{DPI})$ 

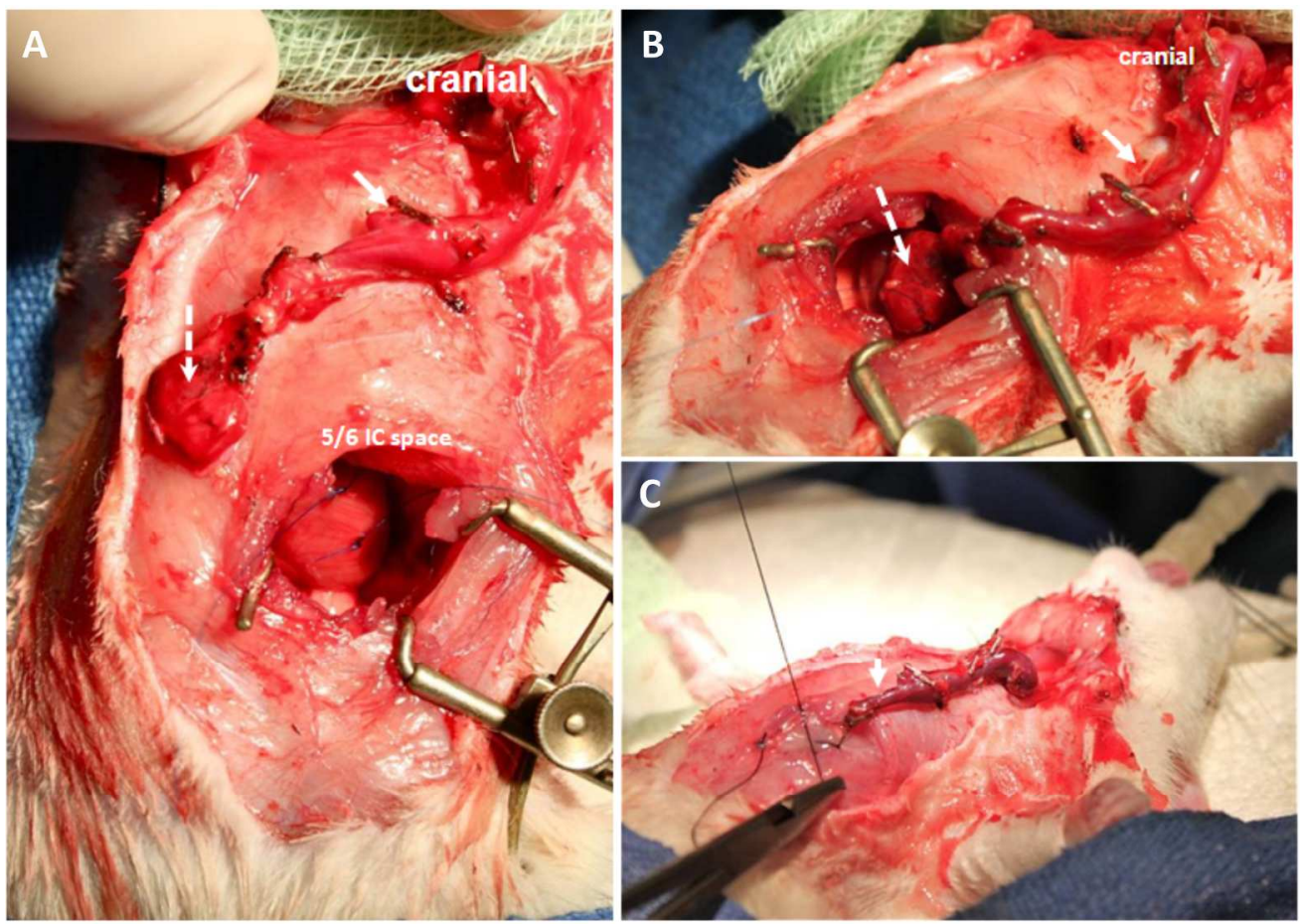

Figure 3 (A) Cardiac muscle flap (dashed arrow) shown to lie over the chest wall with a thoracotomy window that exposed the anterior surface of the recipient's heart, (B) sutured to the heart through a thoracotomy window (note: a segment of the rib was resected to allow the entrance of the pedicle) and (C) The pedicle (solid arrow) ran from the neck vessels, through the split pectoral muscles into the thoracotomy window. 'Cranial' was marked to show orientation. IC=intercostal.

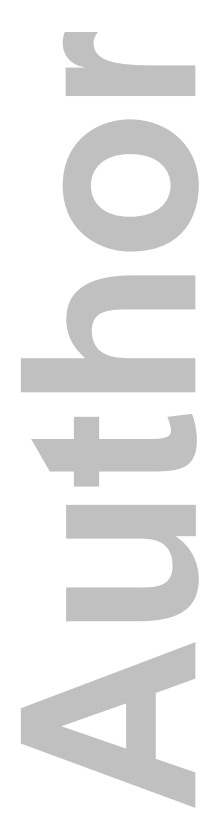

$192 \times 136 \mathrm{~mm}(300 \times 300 \mathrm{DPI})$ 


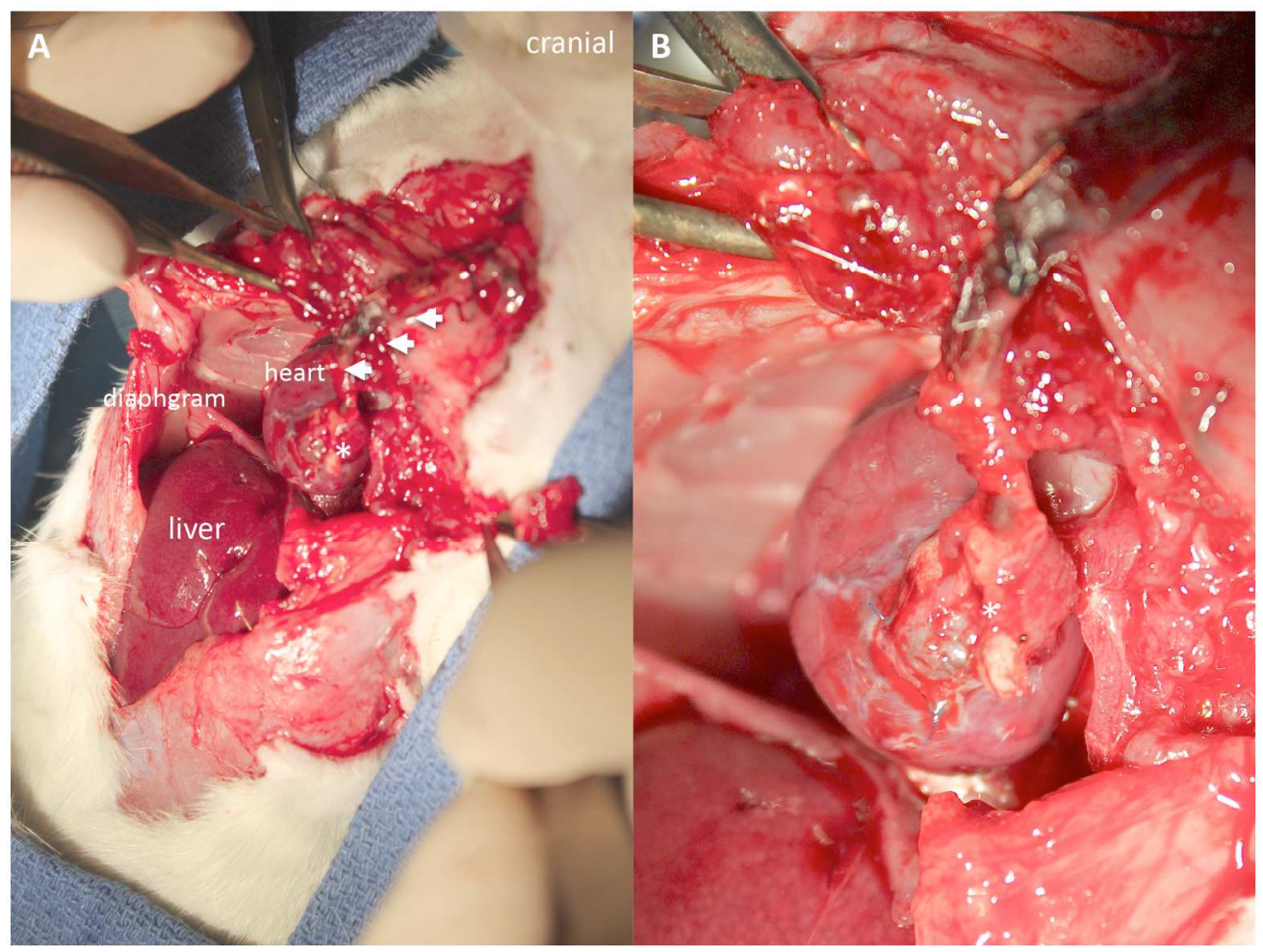

Figure 4 (A) Diaphragmatic approach to harvest the flap $(*)$ \& heart to avoid damage to the pedicle (arrows) prior to the harvest (B) Macroscopic appearance of flap attached to the anterior surface of the ventricle.

$186 \times 140 \mathrm{~mm}(300 \times 300 \mathrm{DPI})$ 


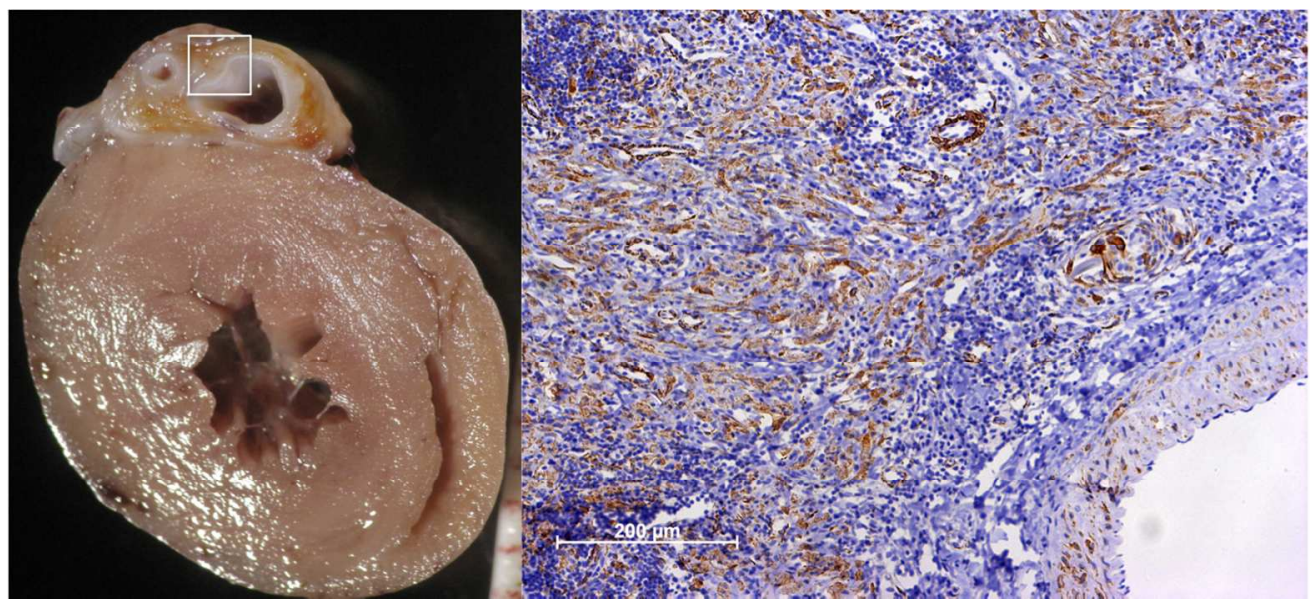

Figure 5 Macroscopic section of harvested specimen (left) showed cross section of transplanted flap on heart surface, with the two lumens being the artery (smaller) and vein (larger) of the arteriovenous loop.

Micrograph sections (right) showed 10x magnification desmin immunostained cardiomyocytes assembled close to the lumen of the flap that remained viable after transplantation. (scale bar $=200 \mu \mathrm{m}$ )

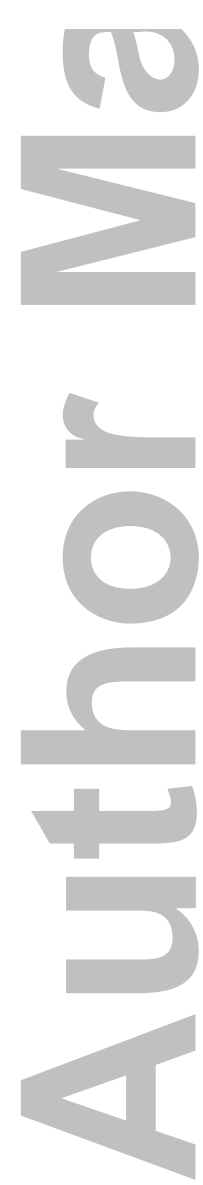

$237 \times 107 \mathrm{~mm}(150 \times 150 \mathrm{DPI})$ 


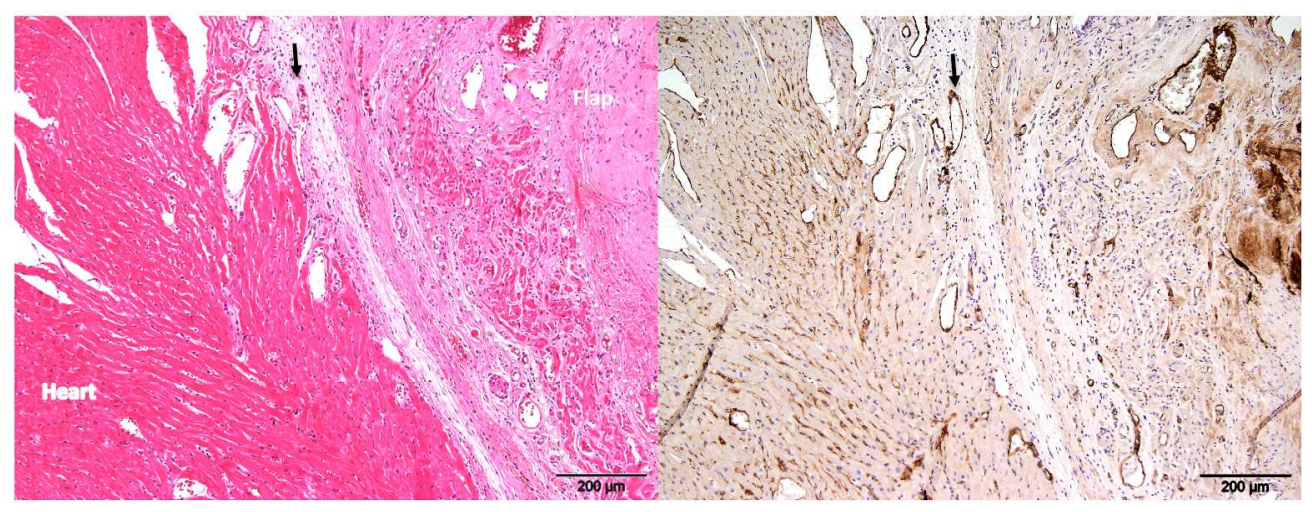

Figure 6 Representative micrographs from consecutive sections at the region of contact between the cardiac muscle flap and the rat heart (left) showed cardiac muscle tissue in the patch (dotted line) separated from the host myocardium by integrated connective tissue of the epicardium and the flap capsule. Picture (right) showed VWF immunostained vessel (arrow) coursed in the epicardium and into the connective tissue between the flap and the heart's surface. $v W F=v o n$ Willebrand factor. (scale bar $=200 \mu \mathrm{m}$ )

$236 \times 90 \mathrm{~mm}(300 \times 300 \mathrm{DPI})$

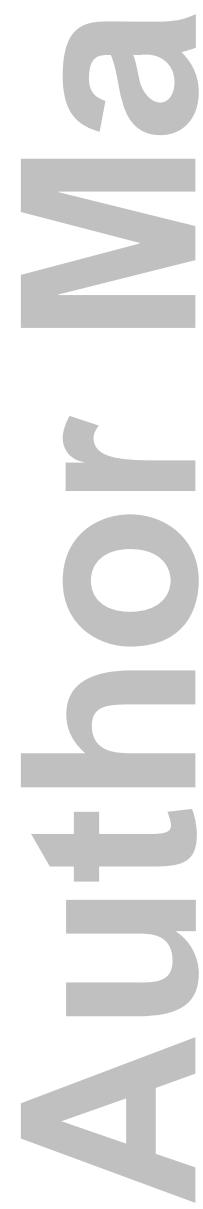




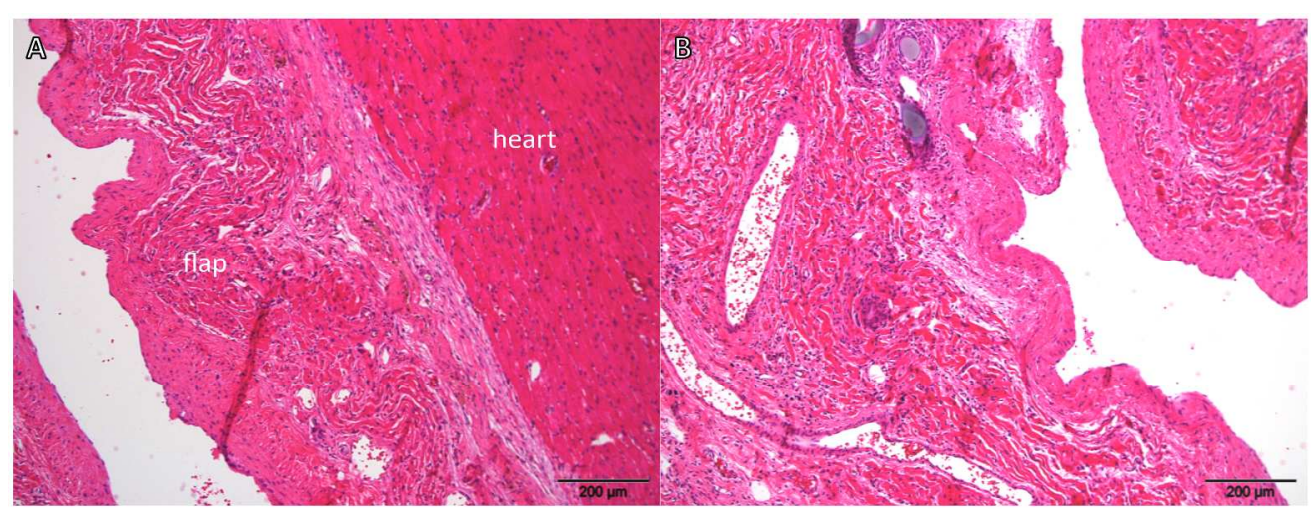

Figure 7 Representative micrographs of H\&E staining showed no demonstrable immune response at $(A)$ the

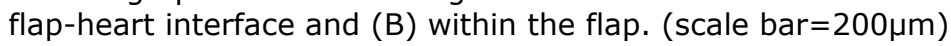

$250 \times 95 \mathrm{~mm}(300 \times 300 \mathrm{DPI})$ 


\section{University Library}

\section{- M M I N E R VA A gateway to Melbourne's research publications}

Minerva Access is the Institutional Repository of The University of Melbourne

Author/s:

Tee, R;Morrison, WA;Dilley, RJ

Title:

A novel microsurgical rodent model for the transplantation of engineered cardiac muscle flap

Date:

2018-07-01

Citation:

Tee, R., Morrison, W. A. \& Dilley, R. J. (2018). A novel microsurgical rodent model for the transplantation of engineered cardiac muscle flap. MICROSURGERY, 38 (5), pp.544-552. https://doi.org/10.1002/micr.30325.

Persistent Link:

http://hdl.handle.net/11343/283836 